ВНУТРИСОСУАИСТАЯ ВИЗУААИЗАЦИЯ

АТЕРОСКАЕРОТИЧЕСКИХ БАЯШЕК У БОАЬНЫХ

С КАРАИОРЕНААЬНЫМ СИНАРОМОМ: ВОЗМОЖНОСТИ ОПТИЧЕСКОЙ КОГЕРЕНТНОЙ ТОМОГРАФИИ

\author{
А.В. Созыкин ${ }^{1,2}$, О.П. Шевченко ${ }^{3}$, Я.А. Наумов ${ }^{1,2}$, А.Г. Строков ${ }^{3}$, В.П. Васильева ${ }^{1,2}$, \\ A.О. Шевченко ${ }^{1,3}$ \\ ' ФГАОУ ВО «РОсСИйский национальный исслеАОвательский меАицинский университет \\ имени Н.И. Пирогова» МинзАрава России, Москва, Российская Федерация \\ 2 ФГБУз «Центральная кАиническая больница Российской акалемии наук», Москва, \\ Российская Фелерация \\ ${ }^{3}$ ФГБУ «Национальный меАицинский исслеАОвательский центр трансплантологии и искусственных \\ органов имени акалемика В.И. Шумакова» Минздрава России, Москва, Российская Фелерация
}

\begin{abstract}
В настоящее время трансплантация почек и применение гемодиализа являются основными методами лечения терминальной почечной недостаточности. При этом главной проблемой при лечении указанной категории пациентов остается высокая смертность, в структуре которой первое место занимают сердечнососудистые заболевания. Показано, что у пациентов с терминальной стадией хронической болезни почек, находящихся на гемодиализе, риск смертности от сердечно-сосудистых заболеваний до 20 раз выше, чем в популяции соответствующего пола и возраста. Указанные данные обусловили целесообразность выделения кардиоренальных взаимоотношений в единый кардиоренальный синдром (КРС). В связи с вышеуказанным особую значимость приобретают методы внутрисосудистой визуализации поражений коронарного русла, в частности оптическая когерентная томография (ОКТ). В настоящем обзоре представлен анализ опубликованных к настоящему времени данных об особенностях и возможностях ОКТ у больных с КРС.
\end{abstract}

Ключевые слова: кардиоренальный синдром, оптическая когерентная томография,

внутрисосудистая визуализачия.

\title{
INTRAVASCULAR IMAGING OF ATHEROSCLEROTIC PLAQUES IN PATIENTS WITH CARDIORENAL SYNDROME: POSSIBILITIES OF OPTICAL COHERENCE TOMOGRAPHY
}

\author{
A.V. Sozykin ${ }^{1,2}$, O.P. Shevchenko ${ }^{3}$, Ya.A. Naumov ${ }^{1,2}$, A.G. Strokov ${ }^{3}$, V.P. Vasilieval,, \\ A.O. Shevchenko ${ }^{1,3}$ \\ ' Pirogov Russian National Research Medical University, Moscow, Russian Federation \\ ${ }^{2}$ Central Clinical Hospital of the Russian Academy of Sciences, Moscow, Russian Federation \\ ${ }^{3}$ Shumakov National Medical Research Center of Transplantology and Artificial Organs, Moscow, \\ Russian Federation
}

\begin{abstract}
Currently, kidney transplantation and the use of hemodialysis are the main methods of treatment of end-stage renal disease. The main problem in the treatment of this category of patients remains high mortality, in the structure of which the first place is occupied by cardiovascular diseases. It is known that cardiovascular mortality risk in patients with hemodialysis-dependent end-stage chronic kidney disease up to 20 times higher than in the sex- and age-matched general population. These data determined isolating of cardiorenal relationships into a cardiorenal syndrome (CRS). It must be confessed that methods of intravascular visualization, particularly optical coherence
\end{abstract}

Для корреспонденции: Наумов Ярослав Анатольевич. Адрес: 117997, Москва, ул. Островитянова, д. 1.

Тел. (915) 164-37-80. E-mail: yaroslavnaumov14@yandex.ru

For correspondence: Naumov Yaroslav Anatol'evich. Address: 1, Ostrovitianova str., Moscow, 117997, Russian Federation.

Tel. (915) 164-37-80. E-mail: yaroslavnaumov14@yandex.ru 
tomography (OCT), are extremely important in diagnostic of coronary artery lesions. This review is devoted to analysis of the published data of the features and capabilities of OCT in patients with the CRS.

Key words: cardiorenal syndrome, optical coherence tomography, intravascular imaging.

Сердечно-сосудистые заболевания являются ведущей причиной заболеваемости и смертности у пациентов с хронической болезнью почек (ХБП). При этом риск смертности возрастает по мере прогрессирования снижения скорости клубочковой фильтрации (СКФ): у пациентов с терминальной стадией ХБП, находящихся на гемодиализе, риск смертности от сердечно-сосудистых заболеваний до 20 раз выше, чем в популяции соответствующего пола и возраста [1]. Тесная взаимосвязь механизмов регуляции и функционирования сердца и почек и нарушений функции того и другого органа послужили основанием выделения кардиоренальных взаимоотношений в единый синдром. Согласно определению Ronco et al. (2008 г.), кардиоренальный синдром - это одновременное нарушение функции сердца и почек, при котором острое или хроническое повреждение одного органа может вызвать острое или хроническое повреждение другого органа [2].

В структуре заболеваемости и смертности больных с кардиоренальным синдромом ведущее место принадлежит ишемической болезни сердца (ИБС). Показано, что ХБП - фактор риска развития острого коронарного синдрома (ОКС) [3].

При аутопсии и прижизненной визуализации методом компьютерной томографии и внутрисосудистого ультразвукового исследования (ВСУЗИ) показана достоверная связь между хронической болезнью почек и тяжестью ишемической болезни сердца и кальцификацией атеросклеротических бляшек в коронарных артериях.

Оптическая когерентная томография позволяет визуализировать атеросклеротические бляшки в коронарных артериях с более высоким разрешением; лучше, чем при ВСУЗИ, определяется пенетрация кальцием. Однако применение ОКТ у пациентов с ХБП ограничено из-за потребности в дополнительном использовании контрастных средств, необходимых для создания оптически прозрачной среды.

В настоящем обзоре представлен анализ опубликованных к настоящему времени данных об особенностях и возможностях ОКТ у больных с КРС. В качестве иллюстраций приведены собственные примеры визуализации атеросклеротического поражения коронарных артерий [4].

Оптическая когерентная томография - это метод внутрисосудистой визуализации, в основе которого лежит принцип отражения лучей инфракрасного диапазона от структур стенки сосуда $[5,6]$. ОКТ была разработана в конце 80 -х - начале 90 -х годов XX века [7]. В начале 2000-х появились исследо- вания, показавшие, что ОКТ является безопасным диагностическим методом и по эффективности не уступает ВСУЗИ [8], что привело к увеличению количества исследований, проводимых с помощью ОКТ и необходимости унификации методик анализа изображений. В 2010 г. была опубликована первая часть обзорного документа по методологии, терминологии и клиническому использованию ОКТ, подготовленная международной группой экспертов [9]. В документе освещались физические принципы ОКТ, методика получения ОКТ-изображений, безопасность и эффективность ОКТ, были представлены данные о нормальной морфологии коронарных артерий, оценке атеросклеротического поражения коронарных артерий, проведен анализ некоторых противоречивых аспектов, а также преимуществ и недостатков ОКТ по сравнению с ВСУЗИ. В 2012 г. опубликована вторая часть обзорного документа, которая была посвящена уточнению некоторых вопросов, не освещенных в первой части, а также описанию методики установки стентов под контролем ОКТ [10]. В 2018 г. была опубликована первая часть согласительного документа Европейской ассоциации по чрескожным коронарным вмешательствам (ЧКВ), в котором проанализированы преимущества и недостатки ВСУЗИ и ОКТ, приведена имеющаяся доказательная база для применения внутрисосудистых методов визуализации, подробно описана техника проведения вмешательств, получения и анализа изображений, а также показания и противопоказания для проведения ВСУЗИ и ОКТ [11]. Вероятно, будет опубликована и вторая часть согласительного документа, поскольку ОКТ сейчас активно развивается и позволяет оценивать многие параметры морфологии коронарных артерий до и после установки стентов и скаффолдов. Считается, что из всех существующих в настоящее время методик внутрисосудистой визуализации (спектроскопия в ближней инфракрасной области, near-infrared spectroscopy - NIRS, ВСУЗИ, ВСУЗИ с виртуальной гистологией, іМАР-ВСУЗИ) ОКТ обладает наибольшей диагностической ценностью [4].

Одна из основных проблем при кардиоренальном синдроме - высокая смертность от сердечнососудистых заболеваний, в структуре которой первое место занимает ИБС $[12,13]$. Вышеуказанные факты обосновывают применение ОКТ при КРС для обнаружения уязвимых бляшек, которые склонны к нарушению целостности покрышки, приводящему к тромбозу, и как следствие - к ОКС. Желательно выявлять такие бляшки в стабильном состоянии пациента. 

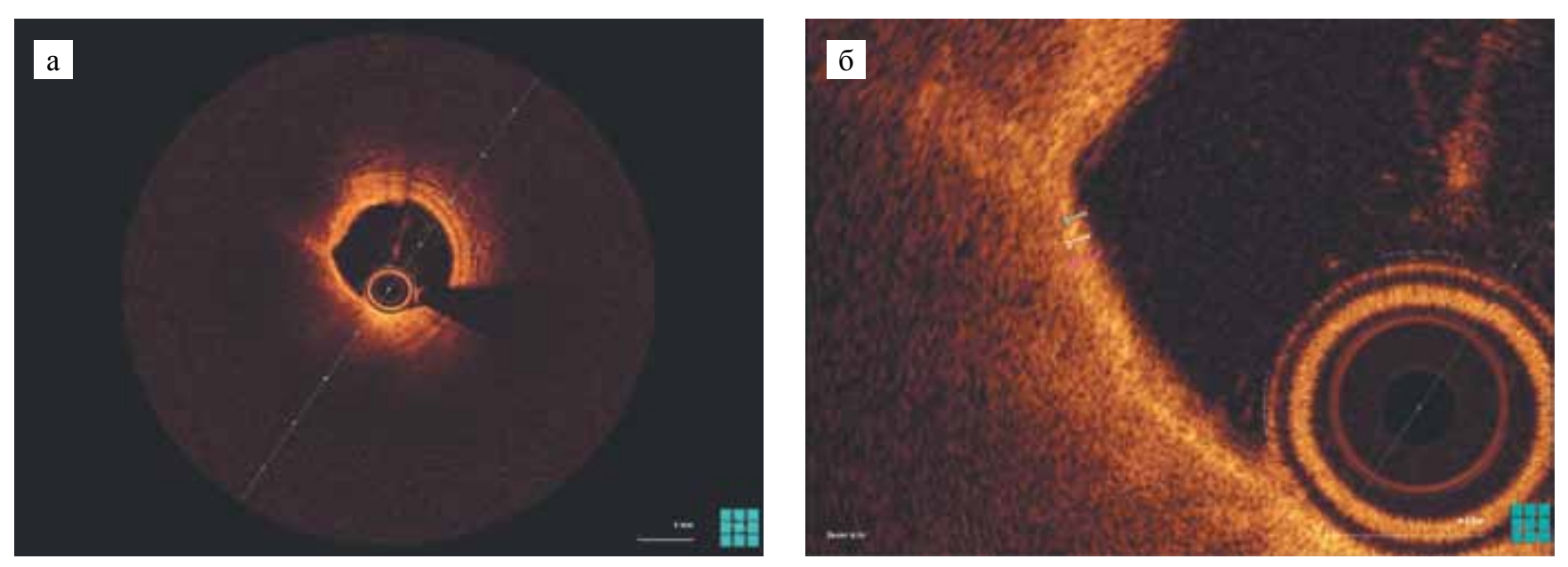

Рис. 1. Атеросклеротическая бляшка с тонкой покрышкой (ОКТ): а - между 6 и 11 часами условного циферблата определяется скопление липидов (липиды занимают более 1 квадранта, что позволяет отнести атеросклеротическую бляшку к богатым липидами); б - толщина покрышки бляшки в самом тонком месте при трехкратном измерении (60 мкм; 60 мкм; 70 мкм; среднее значение - $(60+60+70) / 3=63,3$ мкм)

Fig. 1. Thin-cap fibroatheroma (OCT): a - lipid pool located at 6-11 o'clock (lipids occupy more than 1 quadrant, what means that this plaque is lipid-rich); $\sigma$ - three times measured thickness of the plaque in the thinnest place $(60 \mu \mathrm{m} ; 60 \mu \mathrm{m} ; 70 \mu \mathrm{m}$; average $-(60+60+70) / 3=63.3 \mu \mathrm{m})$

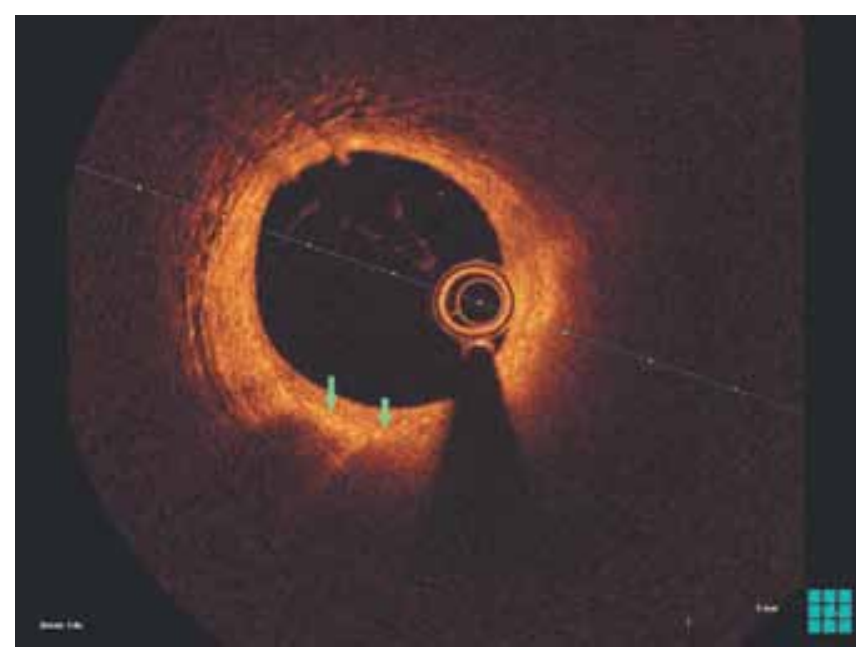

Рис. 2. Скопления макрофагов в атеросклеротической бляшке (ОКТ). Зелеными стрелками отмечены скопления макрофагов: линейные области высокой интенсивности, позади которых видна «тень»

Fig. 2. Cluster of foam cells (OCT), marked by green arrows: linear regions with high intensity with a «shadow» behind

ОКТ - единственный метод внутрисосудистой визуализации, осевое разрешение которого позволяет оценить толщину покрышки бляшки (рис. 1, б) [14], на которую может влиять прием статинов [15]. Статины также обладают плейотропным действием, которое проявляется, в частности, в снижении выраженности макрофагального воспаления (рис. 2) [16, 17].

Однако оценки толщины покрышки недостаточно для отнесения бляшки к бляшкам с тонкой покрышкой, поскольку необходимо оценить также и объем липидного ядра (рис. 1, a). Xing et al. продемонстрировали, что наличие бляшек, богатых липидами, вдвое увеличивает риск развития неблагоприятных событий [18].

Наличие vasa vasorum (рис. 3) и кристаллов холестерина (рис. 4) в бляшке увеличивает вероятность ее разрыва. Чувствительность и специфичность ОКТ по выявлению неоваскуляризации по сравнению с патологоанатомическими данными составляет 52 и 68\% соответственно [19]. Nakamura et al. показали, что у пациентов с наличием кристаллов холестерина в бляшках чаще выявляются бляшки, богатые липидами [20]. Dai et al. продемонстрировали, что кристаллы холестерина чаще встречаются у пациентов с ОИМ с подъемом сегмента ST по сравнению с пациентами с ОКС без подъема сегмента ST (50,8 и 34,7\% соответственно) [21].

Эрозия бляшки является причиной внезапной сердечной смерти приблизительно в 30-40\% случаев $[22,23]$. Показано, что эрозии бляшек чаще выявляются у пациентов моложе 50 лет и чаще расположены в левой коронарной артерии (ЛКА). В исследовании была выявлена связь наличия эрозий бляшек с наличием ХБП. Также предложена классификация факторов риска развития эрозии, которая учитывает как клинические данные, так и данные, которые могут быть получены только при помощи ОКТ, такие как наличие тонкой покрышки [24].

Одним из механизмов развития ОКС считается наличие кальцинатов (рис. 5). У пациентов даже с начальными признаками ХБП чаще выявляются кальцинаты по сравнению с популяцией в целом. Кальциноз становится все более выраженным по 


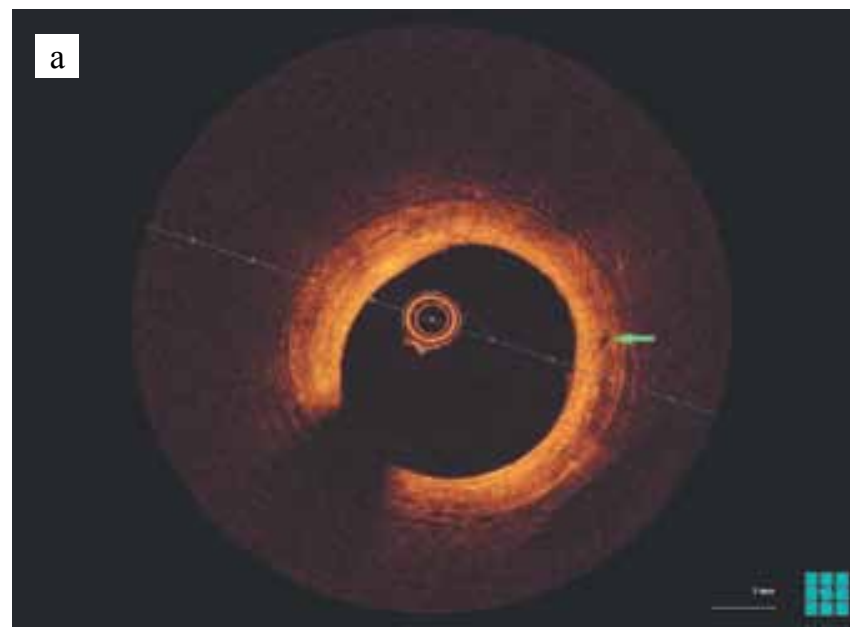

\section{б}

Рис. 3. Микроканалы в атеросклеротической бляшке (отмечены зеленой стрелкой). ОКТ

Fig. 3. Microchannels inside the plaque (marked by green arrow). OCT

мере прогрессирования снижения функции почек, и его наличие независимо ассоциировано с сердечнососудистой смертностью. Максимально выраженный кальциноз наблюдается у пациентов с терминальной почечной недостаточностью (ТПН) [25]. Продемонстрировано, что количество кальцинатов, искажающих просвет сосуда, выше у пациентов с ОИМ с подъемом сегмента ST [26].

Исследования по изучению атеросклеротического поражения коронарных артерий у пациентов с ХБП немногочисленны и гетерогенны. Опубликованные к настоящему времени исследования значительно отличаются по методологии и критериям включения пациентов, поэтому результаты этих исследований также имеют отличия [11, 27, 28].

Kato et al. провели исследование морфологических характеристик атеросклеротических бляшек в коронарных артериях с помощью ОКТ среди пациентов с ХБП и без ХБП. Наличие ХБП устанавливалось, если СКФ, рассчитанная по формуле MDRD,

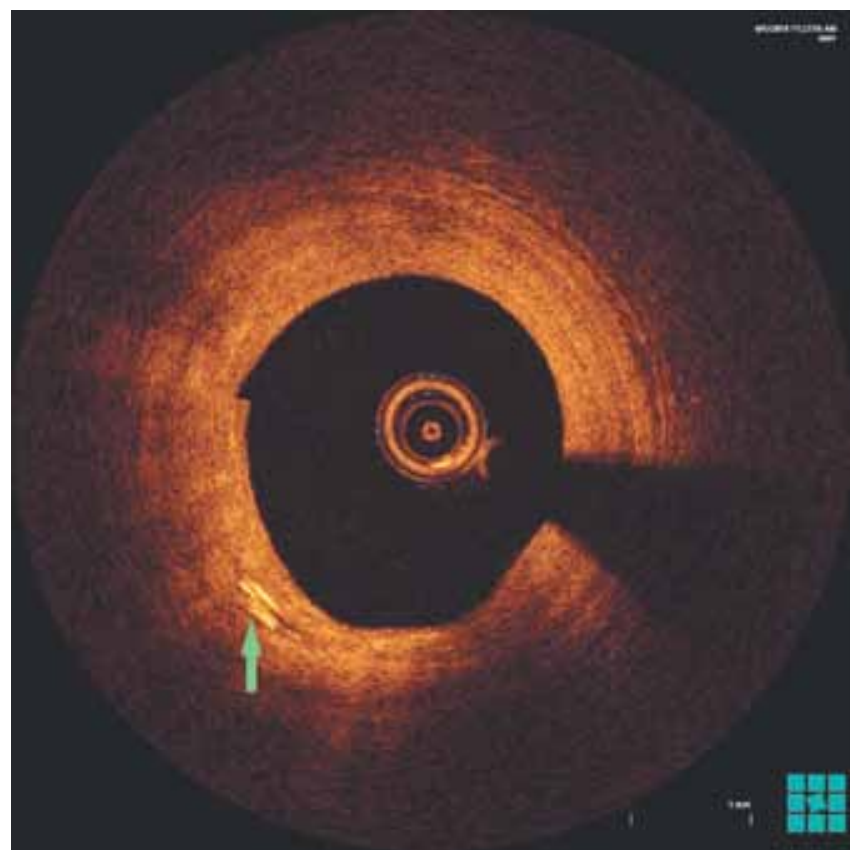

Рис. 4. Кристаллы холестерина в бляшке (отмечены зеленой стрелкой). ОКТ

Fig. 4. Cholesterol crystals inside the plaque (marked by green arrow). OCT

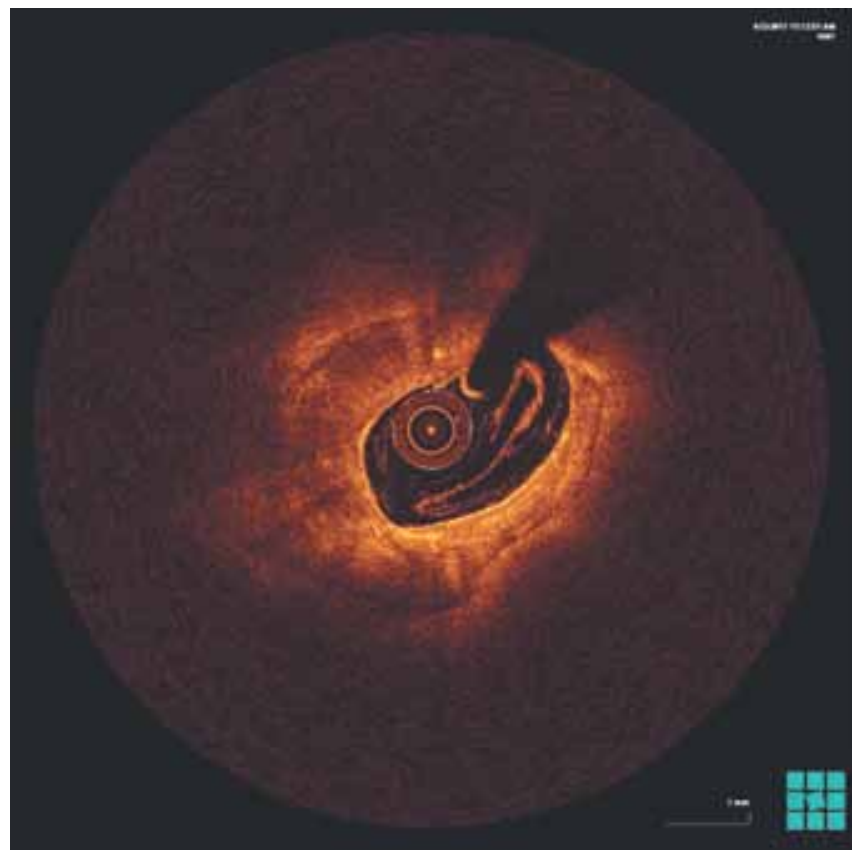

Рис. 5. Кальцинаты в атеросклеротической бляшке (ОКТ). Кальцинаты определяются вдоль всей окружности просвета сосуда (в 4 квадрантах)

Fig. 5. Calcified nodule inside the plaque (OCT). Calcified nodule can be determined along the all circumference of the vessel lumen (4 quadrants)

была $<60$ мл/мин/1,73 м². Если хотя бы на одном из фреймов липидное ядро занимало $\geq 90^{\circ}$, такие бляшки считались бляшками, богатыми липидами. Для 
бляшек, богатых липидами, через каждый 1 мм измерялась величина дуги липидного ядра, затем подсчитывалось среднее значение. Кроме того, были измерены длина липидного ядра, липидный индекс (произведение средней величины дуги липидного ядра и длины липидного ядра, мм), толщина покрышки. Авторы также изучали наличие бляшек с тонкой покрышкой (бляшка, богатая липидами, с толщиной покрышки в самом тонком месте $<65$ мкм), кальцинатов, макрофагов, кристаллов холестерина, микроканалов, бляшек с разрывом и тромбов (с разделением на «белые» и «красные»). Показано, что бляшки у пациентов с ХБП по сравнению с бляшками у пациентов без ХБП имели бо́льшую величину дуги липидного ядра, бо́льшую длину липидов и бо́льший липидный индекс. Кроме того, у пациентов с ХБП чаще встречалась кальцинаты, кристаллы холестерина и разрывы бляшек. Количество бляшек, богатых липидами, бляшек с тонкой покрышкой, макрофагов, микроканалов и тромбов не различалось между группами. Снижение СКФ и наличие СД были независимо связаны с повышением величины липидного индекса [29].

B исследовании Dai et al. снижение функции почек определялось по изменению уровня СКФ. Для расчета использовались уровень креатинина, уровень цистатина $\mathrm{C}$ и оба этих показателя. Пациенты были разделены на три группы согласно уровню СКФ: 1-ю группу составили пациенты с СКФ $\geq 90$ мл/ мин/1,73 м² 2-ю - с СКФ 60-89 мл/мин/1,73 м², 3-юс СКФ $<60$ мл/мин/1,73 м². Толщина покрышки различалась во всех группах пациентов. Средняя величина дуги липидного ядра, длина липидного ядра и липидный индекс различались только при сравнении групп 1 и 2 , а также групп 1 и 3 . Количество бляшек с кристаллами холестерина достоверно различалось только при сравнении групп 1 и 2. Количество бляшек с кальцификацией достоверно различалось только при сравнении групп 1 и 3 [30].

$\mathrm{B}$ исследовании Chin et al. получавшим гемодиализ пациентам, которым проводилось ОКТ-исследование, были подобраны пары среди пациентов без ХБП. Отсутствие ХБП устанавливалось при уровне СКФ $>60$ мл/мин/1,73 м $^{2}$, а также отсутствии каких-либо признаков повреждения почек. При анализе «виновных» бляшек основная и контрольная группы пациентов различались по уровню средней и уровню максимальной величины дуги кальцинатов. При анализе «невиновных» бляшек основная и контрольная группы пациентов также различались по уровню средней и уровню максимальной величины дуги кальцинатов. Авторы провели дополнительный анализ основной группы пациентов, разделив последних на терцили по длительности гемодиализа. При анализе «виновных» бляшек подгруппы пациен- тов различались по уровню средней и максимальной величины дуги кальцинатов и по числу кальцинатов, покрытых тонкой покрышкой, которые определялись как кальцинаты с величиной дуги $>30^{\circ}$, покрытые слоем интимы $<0,5$ мм. При анализе «невиновных» бляшек подгруппы пациентов различались только по уровню средней величины дуги кальцинатов [27].

Sugiyama et al. изучили морфологические характеристики нативных бляшек у пациентов, разделенных на 3 группы согласно СКФ, рассчитанной по формуле, адаптированной для населения Японии: группа без ХБП (СКФ $\geq 60$ мл/мин/1,73 м²), группа с ХБП $(15 \leq$ СКФ $<60$ мл/мин/1,73 м²) и группа с ТПН (СКФ $<15$ мл/мин/1,73 м² и/или гемодиализ). Группы с ХБП и без ХБП для подсчета некоторых параметров были объединены в группу «без ТПН». У пациентов с ХБП по сравнению с пациентами без ХБП отмечалась бо́льшая величина дуги липидного ядра, бо́льшая длина липидов и более высокая частота встречаемости бляшек, богатых липидами. У пациентов с ТПН по сравнению с пациентами без ТПН отмечалась меньшая толщина покрышки бляшки, более высокая частота разрывов бляшек, бо́льшая величина дуги кальцинатов и меньшая толщина покрышки над кальцинатами. Возраст, наличие СД и получение гемодиализа, но не СКФ, были независимо связано с наличием кальцинированных бляшек [31].

Minami et al. исследовали 140 «невиновных» бляшек у 84 пациентов с ИБС, получавших статины, которым было проведено два ОКТ-исследования: при первом поступлении и через 6 мес. Критерием ответа на терапию статинами была выбрана площадь истончения покрышки (области с толщиной покрышки менее 200 мкм). Наличие ХБП определялось по уровню СКФ, рассчитанной по формуле MDRD, $<60$ мл/мин/1,73 м². По сравнению с исходной ОКТ при проведении ОКТ-контроля изменились следующие показатели: уменьшилась площадь истончения покрышки, средняя величина дуги липидного ядра, максимальная величина дуги липидного ядра, длина липидного ядра, липидный индекс, величина стеноза по площади и количество макрофагов; толщина покрышки в самом тонком месте увеличилась. У пациентов с большей площадью истончения покрышки по данным исходной ОКТ отмечалось более значительное снижение площади истончения покрышки при ОКТ-контроле. Более значительное снижение площади истончения покрышки при ОКТ-контроле отмечалось и среди пациентов, ранее не принимавших статины, по сравнению с теми, кто исходно принимал статины. Наличие ХБП являлось неблагоприятным фактором ответа на терапию статинами, а поступление с ОКС во время первой госпитализации - благоприятным [32]. 
У пациентов с ХБП IV-V стадии чаще выявляются многососудистые поражения и наличие бляшек с повышенной толщиной медии, активированными макрофагами и выраженным кальцинозом [33]. Большинство из указанных факторов может быть выявлено с помощью ОКТ, однако у таких пациентов особенно велик риск развития контраст-индуцированной нефропатии. Для решения указанной проблемы применяют кристаллоидные или коллоидные растворы (либо их смеси) вместо рентгеноконтрастного вещества, что позволяет избежать ухудшения функции почек. Karimi Galougahi et al. опубликовали клинический случай, когда пациенту с ХБП V стадии (креатинин 4,5 мг/дл (397,8 мкмоль/л), СКФ 13 мл/мин/1,73 м²), не получавшему гемодиализ, была успешно произведена ОКТ ЛКА. Оптически прозрачную среду создавали с помощью смеси физиологического и коллоидного растворов. После проведения ОКТ функция почек осталась стабильной [34]. К сожалению, работа Karimi Galougahi et al. содержит ограниченные сведения об особенностях атеросклеротического поражения коронарных артерий у данного пациента и применявшегося раствоpa. Azzalini et al. опубликовали подробное описание клинического случая, когда пациенту с СД и ХБП IV стадии (СКФ 16 мл/мин/1,73 м²) была успешно произведена ОКТ ЛКА и диагональной артерии. Оптически прозрачную среду создавали с помощью коллоидного раствора «Декстран 40» со скоростью инфузии 4,0 мл/мин, общее количество введенного раствора составило 14,0 мл. За время госпитализации пациента функция почек оставалась стабильной [35]. Koga et al. опубликовали клинический случай, когда у пациентки, получавшей гемодиализ, визуализировали кальцинированный тромб in vivo с помощью ОКТ, ВСУЗИ и ангиоскопии. Авторы не указали, какой раствор применялся для создания оптически прозрачной среды [36]. Ozaki et al. провели исследование 25 стентированных сегментов у 22 пациентов. Каждому пациенту выполнялась ОКТ с применением контрастного вещества и ОКТ с применением смеси декстрана 40 и лактата Рингера. Было продемонстрировано, что число сегментов, доступных для анализа, не различалось между ОКТ с применением контрастного вещества и ОКТ с применением смеси декстрана 40 и лактата Рингера (97,9 и 96,5\% соответственно) [37].

\section{ЗАКАЮЧЕНИЕ}

Проведение ОКТ у пациентов с нарушением функции почек позволяет точнее оценить морфологию бляшек, выраженность воспаления и «уязвимость» бляшек в целом. ОКТ позволяет четко визуализировать кальциноз и оценить степень его выраженности с высокой точностью. Только ОКТ позволяет увидеть наличие эрозии бляшки и отличить эрозию от разрыва бляшки, что может иметь значение для определения дальнейшей тактики ведения пациента, а именно целесообразности проведения реваскуляризации и в случае положительного решения - оптимального выбора стента или скаффолда. Имеются серьезные ограничения по применению ОКТ у пациентов с ХБП в связи с угрозой развития контраст-индуцированной нефропатии. Как следствие, необходимо дальнейшее совершенствование техники замещения крови оптически прозрачной средой. Одной из актуальных задач является проведение масштабных многоцентровых исследований с целью уточнения возможности выявления уязвимых атеросклеротических бляшек при рутинном применении ОКТ и возможности снижения риска ОКС, а также влияния на выживаемость пациентов без нежелательных событий как одну из основополагающих целей любого вмешательства.

Авторы заявляют об отсутствии конфликта интересов.

The authors declare no conflict of interest.

\section{СПИСОК АИТЕРАТУРЫ / REFERENCES}

1. Hou F, Jiang J, Chen J, Yu X, Zhou Q, Chen P et al. China collaborative study on dialysis: a multi-centers cohort study on cardiovascular diseases in patients on maintenance dialysis. BioMed Central nephrology. 2012 Aug 30; 13: 94. doi: 10.1186/1471-2369-13-94.

2. Ronco C, House AA, Haapio M. Cardiorenal syndrome: refining the definition of a complex symbiosis gone wrong. Intensive care medicine. 2008 May; 34 (5): 957962. doi: 10.1007/s00134-008-1017-8. Epub 2008 Feb 5.

3. Uemura S, Soeda T, Sugawara Y, Ueda T, Watanabe M, Saito $Y$. Assessment of Coronary Plaque Vulnerability with Optical Coherence Tomography. Acta Cardiologica Sinica. 2014 Jan; 30 (1): 1-9.

4. Созыкин АВ, Никитин АЭ, Шлыков АВ, Новикова НА, Кузьмина ИВ, Эртман ВГ и др. Поражение ствола левой коронарной артерии при стабильной ишемической болезни сердца: возможности оптической когерентной томографии в выборе врачебной тактики и оптимизации интервенционного лечения. Эндоваскулярная хирургия. 2018; 5 (4): 402-409. Sozykin AV, Nikitin AE, Shlykov AV, Novikova NA, Kuz'mina IV, Ertman $V G$ et al. Left main coronary artery disease: opportunities of optical coherence tomography in the choice of treatment strategy and optimization of percutaneous coronary interventions. Russian Journal of Endovascular Surgery. 2018; 5 (4): 402-409. doi: 10.24183/24094080-2018-5-4-402-409.

5. Kume T, Uemura S. Current clinical applications of coronary optical coherence tomography. Cardiovascular intervention and therapeutics. 2018 Jan; 33 (1): 1-10. doi: 10.1007/s12928-017-0483-8.

6. Suh WM, Seto AH, Margey RJ, Cruz-Gonzalez I, Jang IK. Intravascular detection of the vulnerable plaque. Circu- 
lation. Cardiovascular imaging. 2011 Mar; 4 (2): 169178. doi: 10.1161/CIRCIMAGING.110.958777.

7. Huang D, Swanson EA, Lin CP, Schuman JS, Stinson $W G$, Chang $W$ et al. Optical coherence tomography. Science. 1991 Nov 22; 254 (5035): 1178-1181.

8. Jang IK, Bouma BE, Kang DH, Park SJ, Park SW, Seung KB et al. Visualization of coronary atherosclerotic plaques in patients using optical coherence tomography: comparison with intravascular ultrasound. Journal of the American College of Cardiology. 2002 Feb 20; 39 (4): 604-609.

9. Prati F, Regar E, Mintz GS, Arbustini E, Di Mario C, Jang IK et al. Expert's OCT Review Document. Expert review document on methodology, terminology, and clinical applications of optical coherence tomography: physical principles, methodology of image acquisition, and clinical application for assessment of coronary arteries and atherosclerosis. European heart journal. 2010 Feb; 31 (4): 401-415. doi: 10.1093/eurheartj/ehp433.

10. Prati F, Guagliumi G, Mintz GS, Costa M, Regar E, Akasaka $T$ et al. Expert's OCT Review Document. Expert review document part 2: methodology, terminology and clinical applications of optical coherence tomography for the assessment of interventional procedures. European heart journal. 2012 Oct; 33 (20): 2513-2520. doi: 10.1093/eurheartj/ehs095.

11. Räber L, Mintz GS, Koskinas KC, Johnson TW, Holm NR, Onuma $Y$ et al. ESC Scientific Document Group. Clinical use of intracoronary imaging. Part 1: guidance and optimization of coronary interventions. An expert consensus document of the European Association of Percutaneous Cardiovascular Interventions. European heart journal. 2018 Sep 14; 39 (35): 3281-3300. doi: 10.1093/ eurheartj/ehy285.

12. Sinclair H, Bourantas C, Bagnall A, Mintz GS, Kunadian $V$. OCT for the identification of vulnerable plaque in acute coronary syndrome. Journal of the American College of Cardiology cardiovascular imaging. 2015 Feb; 8 (2): 198-209. doi: 10.1016/j.jcmg.2014.12.005.

13. Boi A, Jamthikar AD, Saba L, Gupta D, Sharma A, Loi B et al. A survey on coronary atherosclerotic plaque tissue characterization in intravascular optical coherence tomography. Current atherosclerosis reports. 2018 May 21; 20 (7): 33. doi: 10.1007/s11883-018-0736-8.

14. Yonetsu T, Jang IK. Advances in intravascular imaging: new insights into the vulnerable plaque from imaging studies. Korean circulation journal. 2018 Jan; 48 (1): 1-15. doi: 10.4070/kcj.2017.0182.

15. Zheng G, Chen J, Lin C, Huang X, Lin J. Effect of statin therapy on fibrous cap thickness in coronary plaques using optical coherence tomography: a systematic review and meta-analysis. Journal of interventional cardiology. 2015 Dec; 28 (6): 514-522. doi: 10.1111/joic.12245.

16. Komukai K, Kubo T, Kitabata H, Matsuo Y, Ozaki Y, Takarada $S$ et al. Effect of atorvastatin therapy on fibrous cap thickness in coronary atherosclerotic plaque as assessed by optical coherence tomography: the EASYFIT study. Journal of the American College of Cardio- logy. 2014 Dec 2; 64 (21): 2207-2217. doi: 10.1016/j. jacc.2014.08.045.

17. Самойленко ВВ, Шевченко ОП, Буриев ВИ. Применение статинов в периоперационном периоде с позиций доказательной медицины. Рациональная фармакотерапия в кардиологии. 2014; 10 (5): 548-553. Samoylenko VV, Shevchenko OP, Burtsev VI. Statins use in the perioperative period according to the evidencebased medicine. Ration Pharmacother Cardiol. 2014; 10 (5): 548-553.

18. Xing L, Higuma T, Wang Z, Aguirre AD, Mizuno K, Takano $M$ et al. Clinical significance of lipid-rich plaque detected by optical coherence tomography: a 4-year follow-up study. Journal of the American College of Cardiology. 2017 May 23; 69 (20): 2502-2513. doi: 10.1016/j. jacc.2017.03.556.

19. Kume T, Okura H, Yamada R, Koyama T, Fukuhara K, Kawamura A et al. Detection of plaque neovascularization by optical coherence tomography: ex vivo feasibility study and in vivo observation in patients with angina pectoris. The Journal of invasive cardiology. 2016 Jan; 28 (1): 17-22.

20. Nakamura S, Inami S, Murai K, Takano M, Takano $H$, Asai $K$ et al. Relationship between cholesterol crystals and culprit lesion characteristics in patients with stable coronary artery disease: an optical coherence tomography study. Clinical research in cardiology : official journal of the German Cardiac Society. 2014 Dec; 103 (12): 1015-1021. doi: 10.1007/s00392-014-0748-5.

21. Dai J, Tian J, Hou J, Xing L, Liu S, Ma Let al. Association between cholesterol crystals and culprit lesion vulnerability in patients with acute coronary syndrome: An optical coherence tomography study. Atherosclerosis. 2016 Apr; 247: 111-117. doi: 10.1016/j.atherosclerosis.2016.02.010.

22. Arbustini E, Dal Bello B, Morbini P, Burke AP, Bocciarelli $M$, Specchia $G$ et al. Plaque erosion is a major substrate for coronary thrombosis in acute myocardial infarction. Heart. 1999 Sep; 82 (3): 269-272.

23. Virmani R, Kolodgie FD, Burke AP, Farb A, Schwartz SM. Lessons from sudden coronary death: a comprehensive morphological classification scheme for atherosclerotic lesions. Arteriosclerosis, thrombosis, and vascular biology. 2000 May; 20 (5): 1262-1275. doi: 10.1161/01. ATV.20.5.1262.

24. Dai J, Xing L, Jia H, Zhu Y, Zhang S, Hu S et al. In vivo predictors of plaque erosion in patients with ST-segment elevation myocardial infarction: a clinical, angiographical, and intravascular optical coherence tomography study. European heart journal. 2018 Jun 7; 39 (22): 2077 2085. doi: 10.1093/eurheartj/ehy101.

25. Tölle M, Reshetnik A, Schuchardt M, Höhne M, van der Giet $M$. Arteriosclerosis and vascular calcification: causes, clinical assessment and therapy. European journal of clinical investigation. 2015 Sep; 45 (9): 976-985. doi: 10.1111/eci.12493.

26. Ong DS, Lee JS, Soeda T, Higuma T, Minami Y, Wang Z et al. Coronary calcification and plaque vulnerability: an optical coherence tomographic study. Circulation. Car- 
diovascular imaging. 2016 Jan; 9 (1). pii: e003929. doi: 10.1161/CIRCIMAGING.115.003929.

27. Chin CY, Matsumura M, Maehara A, Zhang W, Lee CT, Yamamoto $\mathrm{MH}$ et al. Coronary plaque characteristics in hemodialysis-dependent patients as assessed by optical coherence tomography. The American journal of cardiology. 2017 May 1; 119 (9): 1313-1319. doi: 10.1016/j. amjcard.2017.01.022.

28. Munnur RK, Nerlekar N, Wong DT. Imaging of coronary atherosclerosis in various susceptible groups. Cardiovascular diagnosis and therapy. 2016 Aug; 6 (4): 382-395. doi: 10.21037/cdt.2016.03.02.

29. Kato K, Yonetsu T, Jia H, Abtahian F, Vergallo R, Hu S et $a l$. Nonculprit coronary plaque characteristics of chronic kidney disease. Circulation. Cardiovascular imaging. 2013 May 1; 6 (3): 448-456. doi: 10.1161/CIRCIMAGING.112.000165.

30. Dai J, Xing L, Hou J, Jia H, Hu S, Tian J et al. Chronic kidney disease predicts coronary plaque vulnerability: an optical coherence tomography study. Coronary artery disease. 2017 Mar; 28 (2): 135-144. doi: 10.1097/ MCA. 0000000000000452.

31. Sugiyama T, Kimura S, Ohtani H, Yamakami Y, Kojima K, Sagawa Y et al. Impact of chronic kidney disease stages on atherosclerotic plaque components on optical coherence tomography in patients with coronary artery disease. Cardiovascular intervention and therapeutics. 2017 Jul; 32 (3): 216-224. doi: 10.1007/s12928-0160408-y.

32. Minami $Y$, Wang $Z$, Aguirre AD, Ong DS, Kim CJ, Uemura $S$ et al. Clinical predictors for lack of favorable vascular response to statin therapy in patients with coronary artery disease: a serial optical coherence tomo- graphy study. Journal of the American Heart Association. 2017 Nov 1; 6 (11). pii: e006241. doi: 10.1161/ JAHA.117.006241.

33. Aoki J, Ikari Y. Cardiovascular disease in patients with end-stage renal disease on hemodialysis. Annals of $v a-$ scular diseases. 2017 Dec 25; 10 (4): 327-337. doi: 10.3400/avd.ra.17-00051.

34. Karimi Galougahi K, Zalewski A, Leon MB, Karmpaliotis $D$, Ali ZA. Optical coherence tomography-guided percutaneous coronary intervention in pre-terminal chronic kidney disease with no radio-contrast administration. European heart journal. 2016 Apr 1; 37 (13): 1059. doi: 10.1093/eurheartj/ehv667.

35. Azzalini L, Mitomo S, Hachinohe D, Regazzoli D, Colombo A. Zero-contrast percutaneous coronary intervention guided by dextran-based optical coherence tomography. The Canadian journal of cardiology. 2018 Mar; 34 (3): 342.e1-342.e3. doi: 10.1016/j.cjca.2017.11.008.

36. Koga S, Ikeda S, Nakata T, Kawano H, Abe K, Maemura $K$. Diverse findings in calcified thrombus between histopathology and in vivo imaging including intravascular ultrasound, optical coherence tomography, and angioscopy. International heart journal. 2015; 56 (6): 661-663. doi: 10.1536/ihj.15-117.

37. Ozaki Y, Kitabata H, Tsujioka H, Hosokawa S, Kashiwagi $M$, Ishibashi $K$ et al. Comparison of contrast media and low-molecular-weight dextran for frequency-domain optical coherence tomography. Circulation journal: official journal of the Japanese Circulation Society. 2012; 76 (4): 922-927.

Статья поступила в редакиию 10.10.2019 2. The article was submitted to the journal on 10.10.2019 